\title{
Session V: Lung
}

\section{Natural history of intraepithelial neoplastic lung le- sions: Implication for early detection and chemo- prevention}

\section{Stephen Lam \\ British Columbia Cancer Agency and the University of British Columbia, Vancouver, BC, Canada}

Lung cancer is the most common cause of cancer death world-wide; more than 1.3 million people die of lung cancer annually. Five-year survival rates after lung cancer diagnosis have improved only marginally in the last three decades. While anti-tobacco initiatives in young people are important in preventing lung cancer in the long term, additional measures such as early detection and chemoprevention are needed for individuals already at risk due to tobacco smoke exposure. Though early detection has been shown to be effective in reducing the incidence and mortality of cancer of the cervix, breast, and colon, there is considerable skepticism in applying the same cancer control strategy to lung cancer. The commonest criticism is the uncertain identity of intraepithelial neoplastic lesions (IEN) and the natural history of these lesions.

There are unique challenges in studying IEN lesions in the lung. In contrast to other epithelial organs, the lung is an internal organ consisting of a complex branching system of airways leading to gas exchange units with a surface area the size of a tennis court. Instead of a single cell type, lung cancer consists of several cell types, preferentially located in different parts of the bronchial tree. No single method can scan the entire bronchial epithelium for IEN lesions and allow tissue sampling for pathological diagnosis and molecular profiling. Autofluorescence bronchoscopy is a major advance in detecting high-grade IEN lesions in the central airways and obtaining biopsies. It has contributed to improved histopathological classification and molecular profiling of IEN lesions. However, the biopsy procedure may remove small lesions, giving the impression that the majority of IEN lesions regress spontaneous- ly. Progress has been made in the use of other optical imaging methods, such as confocal micro-endoscopy and optical coherence tomography (OCT) to characterize these lesions without removing them. Due to size limitation for optical probes and a more stringent regulatory approval process for topical application of contrast/labeling agents in the lung, further technology development is needed before these methods can be applied as optical biopsy tools. However, despite current limitations, fluorescence bronchoscopy-directed biopsies taken from the same site before and after progression from low-grade IEN lesions to carcinoma in situ or early invasive cancer can provide an extremely valuable tissue resource for genomic studies to further our understanding of the carcinogenesis process and to identify targets for intervention.

CT of the chest is a sensitive method to detect peripheral IEN lesions beyond the visual range of fiberoptic bronchoscopes. Recent development of navigation systems is a step towards image-guided biopsies of peripheral lung lesions that will lead to a better understanding of progression pathways of non-squamous lesions. Rapid advances in imaging technologies provide unprecedented opportunity to characterize IEN lesions in the lung, study their natural history, and determine their significance as targets for early detection and intervention such as chemoprevention.

\section{New technologies for in vitro lung cancer screening and image-guided interventions}

\section{Eric J. Seibel \\ University of Washington, Technology Development, HIT Lab, Seattle, WA 98195, USA}

An optical projection tomography microscope (OPTM) has been developed to generate 3D images with submicron and isometric resolution of cells and nuclei from cell suspensions injected into a rotating capillary tube for multi-perspective image collection. 
The analogous imaging method is CT, but instead of using $\mathrm{X}$-rays, scanned light is absorbed by hematoxylin nuclear stain. To determine the ability of OPTM to differentiate cultured human normal epithelial nuclei from cultured human lung adenocarcinoma nuclei, an initial classifier was developed based on six 3D features. This pilot 400-cell study has achieved $90 \%$ sensitivity at $95 \%$ specificity. Current work is extending the OPTM 3D imaging to whole cells isolated from sputum samples and fine-needle aspirates.

A scanning fiber endoscope (SFE) has been developed that images in vivo using an ultrathin and flexible catheter-like probe. Red, green, and blue (RGB) laser light $(635,532$ and $440 \mathrm{~nm})$ is combined into a singlemode optical fiber, the distal tip of which is driven at mechanical resonance $(5 \mathrm{KHz})$. This $1.0 \mathrm{~mm}$ diameter fiber scanner generates 500 line images at $15 \mathrm{~Hz}$ by scanning the RGB laser light in a spiral pattern from the distal tip. The backscattered light is collected from the $70^{\circ}$ scanned field by a ring of 12 plastic optical fibers that surround the fiber scanner, making the current prototype $1.6 \mathrm{~mm}$ in diameter. Future prototypes will be $1.0 \mathrm{~mm}$ in diameter by using alternative light collection methods. A custom guidance system which introduces SFE through the working channel of a standard bronchoscope while directed by a guidewire is being tested in pigs. The interactive $3 \mathrm{D}$ user interface has been developed to perform five functions: automated segmentation and virtual rendering of airways from a chest CT scan; virtual bronchoscopic navigation of the airway anatomy and path-planning for biopsy; real-time tracking and display of the endoscope tip using an electromagnetic sensor; cell sampling in the more peripheral lung under direct video imaging; and automated annotation of procedural events, including the 3D path, optical diagnoses, and biopsy locations.

\section{Applications of MALDI-MS imaging to early detec- tion of lung cancer}

\section{Pierre Massion \\ Vanderbilt-Ingram Cancer Center, Cancer Biology, Nashville, TN 37232, USA}

Imaging mass spectrometry allows the analysis of protein expression in tissue sections. This profiling of tissue may lead to analysis of one or more spots from various histologically determined areas of interest. More complete information on the distribution of signals within the tissue is obtained by imaging the tissue through analysis of an array of spots containing hundreds to thousands of pixels from a single biopsy specimen. A mass spectrum can be displayed for each pixel, covering proteins with a molecular weight of a few thousand to more than 100,000. A plot of the relative intensity of any molecular weight species in each pixel over the imaged area produces a molecular weight-specific image of the tissue. Acquiring such data-rich spectral patterns necessitates advanced computational approaches to data mining and interpretation and represents a critical part of the process of discovery of protein signatures. Potential clinical applications of this technology include delimitating tumor margins, and rapid diagnostic evaluation of clinical samples such as fine needle aspirates. We also plan to use this technology to study distribution of proteins, discriminating between stages of preinvasive lesions and predicting lung tumor development.

\section{Solving the lung cancer paradox}

\section{Geoffrey McLennan}

University of Iowa Health Care, Internal Medicine, Iowa City, IA 52242, USA

The paradox of lung cancer, that small peripheral lung cancers can be detected by multidetector computed tomography (MDCT), but not diagnosed easily, remains a substantial clinical problem. The natural history of small peripheral lung cancers remains uncertain and therapeutic options unsatisfactory. Most detected nodules suspicious for lung cancer represent noncancerous disease. Unlike other organs, the lung presents specific imaging problems because of motion associated with breathing and cardiac oscillation in the left lower lung. One further barrier to developing better imaging methods for lung nodules is lack of understanding their pathological structure in 3D related to imaging-based structure and function. Determining how much of a cancer nodule is, in fact, cancer, and how it is organized within a nodule, appears critical for further development of more specific imaging strategies. As part of our efforts to assist with this lung cancer paradox, we have analyzed several human peripheral lung cancers for 3D pathology. Using digital imaging, enabling development of image-based pulmonary pathfinding for placement of submillimeter diameter devices through the bronchial tree to the suspect bronchogenic lesion in full 3D, allows rapid fine manipulation into a precise location within the lesion through 
magnetic guidance; verification of device placement through magnetic tracking, with secondary fluoroscopic verification; macro- or micro-optical sampling of the lesion through confocal or other microscopy; and, if necessary, ablation if the lesion is considered possibly malignant. Magnetic guidance can also stabilize a device for real-time microscopy. These methods have all been developed, and some have been tested, in translational blinded clinical research studies.

\section{Robotically assisted lung biopsy under CT fluo- roscopy}

\section{Kevin Cleary}

Georgetown University Medical Center, ISIS Center, Department of Radiology, Washington, DC, 20007, USA

Our goal to develop a robotic system to assist the physician in accurate CT fluoroscopy-guided needle biopsy of lung nodules consists of two phases. The completed first phase focused on demonstrating the feasibility of using a joystick-controlled robotic "needle driver" to accurately hit simulated lesions in a phantom under CT fluoroscopy guidance. The second phase, currently in progress, is to develop an enhanced gripper along with path planning capability and demonstrate this approach in phantom and animal studies. In this phase, the gripper will be enhanced to incorporate force-sensing capability (automatic release of the needle if a certain force level is exceeded - a safety feature required for clinical application) and a rotating needle (to minimize the force needed for needle placement). This project is a collaboration between the Computer Aided Interventions and Medical Robotics (CAIMR) group at Georgetown University, the Urology Robotics Laboratory at Johns Hopkins Bayview Medical Center, and the Department of Radiology at the University of Maryland Medical Center. Image-guided intervention for stage I lung cancer
and pulmonary nodules

\author{
Robert Suh \\ University of California, Los Angeles, Department \\ of Radiological Sciences, David Geffen School of \\ Medicine at UCLA, Los Angeles, CA 90995, USA
}

Over the prior decade, an increasing number of minimally invasive or IGI techniques have been introduced into clinical practice for both diagnosis and treatment of primary lung malignancy.

Percutaneous transthoracic needle biopsy (TNB) has emerged over the past three decades as a minimally invasive procedure of choice for diagnosis of pulmonary nodules. Improvements in imaging technology, cytopathologic technique, and needle design have rendered TNB safe, inexpensive, reliable, and widely used. Previously inaccessible lesions can now be sampled using cross-sectional imaging, and tissue retrieval sufficient for histologic diagnosis can now be safely performed with currently available small-gauge cutting needles.

A large number of those diagnosed with lung carcinoma each year present with advanced or widespread disease at the time of diagnosis; only approximately $15 \%$ of patients are surgical candidates. Some patients, however, have technically resectable disease but cannot undergo surgical resection because of co-morbid cardiopulmonary disease. This latter patient population represents a suitable target for novel, minimally invasive "lung-sparing" therapies providing local control.

IGI and video-assisted thoracoscopic approaches have become attractive and increasingly used alternatives to open thoracic surgical resection. Minimally invasive therapies have emerged within the last decade that use ablative thermal energy sources including RF, cryoablation, focused ultrasound, laser, and microwave. Of these, RF ablation has been the best developed, secondary to the advent of bipolar and multielectrode and internal tip-cooling RF electrodes, as well as advances in CT technology.

Recent advances in biopsy needle technology are providing interesting results from minimally invasive image-guided therapy for stage I non-small cell lung cancer. 